\title{
Étude expérimentale du déferlement de la houle
}

\section{Experimental study on the breaking of waves}

\author{
PAR F. SUQUET \\ Inglinteur aux ETabissements Neyrpic \\ English synopsis p. 311 \\ Le déferlement de la houle est depuis plusieurs années à \\ l'étude au Laboratoire Dauphinois d'Hydraulique; M. Brésen a \\ présenté sur ce sujet une communication au Comité technique \\ de la Société Hydrotechnique de France, le 4 juin 1948. \\ A la suite de nombreuses demandes qui nous ont été adressées, \\ nous publions aujourdhui dans la Houille Blanche les premiers \\ résultats de nos expériences. Ils doivent ètre considérés comme \\ provisoires et des études actuellement en cours les compléteront \\ sur de nombreux points.
}

\section{INTRODUCTION}

Le problème du déferlement de la houle sur une plage inclinée est un problème encore mal connu et peu étudié.

Il est lié jusqu'à un certain point à un autre problème mieux connu : celui de la forme limite de. la houle en profondeur constante. Cette forme a été étudiée au point de vue théorique par Storks et ses continuateurs et récemment par M. Mrche (1) $\left(^{*}\right)$; ce dernier a montré, de façon générale, que la forme limite d'une houle, irrotationnelle ou non, était symétrique par rapport à la verticale et comportait en crête un point anguleux, l'angle des tangentes en ce point étant de $120^{\circ}$.

Le probleme proprement dit du déferlement sur un plan incliné a été abordé ces temps derniers par divers auteurs qui, sans résoudre le problème complètement, ont cependant ouvert des voies nouvelles très intéressantes. On peut

(*) Les chiffres entre parenthèses renvoient à la Bibliographie. citer ainsi : M. Miche, en France (1), M. SToKER (2) et M. Sverdrup (3) aux Etats-Unis (**).

Les premières observations du phénomène remontent, à notre connaissance, à Bremontrer. Peu d'études expérimentales ont été faites : nous devons cependant citer les essais de M. LARRAS à Alger (4) et ceux de M. G. FErro (5) à l'Université de Padoue, ainsi que les études en laboratoire et les observations à la mer faites aux Etats-Unis et en Angleterre lor's de la dernière guerre, en vue des opérations de débarquement (6) (7).

Le but de nos essais a été principalement de « débrouiller» le problème du déferlement de la houle; nous avons essayé en particulier d'avoir une idée générale du phénomène, et de déterminer l'influence de certains paramètres; enfin nous avons mesuré au point de déferlement certaines grandeurs intéressantes telles que la profondeur.

(*) Nous n'avons pu nous procurer l'ouvrage do M. Bonbr cité en référence par M. Storen. 


\section{PREMIELEE PARTIE}

\section{DISPOSITIF D'ESSAI ET DESCRIPTION DU PHENOMENE}

\section{I. - Dispositif d'essai.}

\section{Canal dessai - Caractéristiques.}

Les essais $1,2,3,4,5,6$ ont été faits sur un canal à houle de $60 \mathrm{~cm}$ de largeur et $20 \mathrm{~m}$ de longueur; la profondeur aux pointes de mesures a toujours été voisine de $60 \mathrm{~cm}$. Des essais systématiques effectués au Laboratoire ont montré que les houles produites sur ce canal avaient des dimensions suffisantes pour qu'on puisse négliger l'effet d'échelle sur le déferlement et l'amortissement propre de la houle. Ce canal ne comportait pas de paroi vitrée.

Le fond incliné était en bois composé de planches jointives et très sensiblement plan; il était maconné sur les bords. Le fond incliné pouvait ainsi être considéré comme pratiquement étanche.

Nous avons essayé sur ce canal quatre pentes diférentes :

$$
\frac{1}{5,7} \quad-\frac{1}{11} \quad \frac{1}{15,5} \quad \frac{1}{25}
$$

Pour les deux dernières pentes, un raccordement de courbure assez forte était ménagé entre le fond horizontal du canal et le début du plan incliné en bois, afin d'éviter de donner à ce dernier une trop grande longuene (fig. 1). Nous reviendrons plus loin sur l'influence de ce raccordement.

Fia. 1

Sur la pente $\frac{1}{25}$ il a été effectué deux séries d'essais : l'une avec la disposition habituelle du plan incliné, l'autre en recouvrant ce dernier de métal déployé afin de modifier sa rugosité.

La houle stait produile par un battear formé d'an volet plan, oscillant autour d'un axe placé an fond du canal. Le canal était écuipe de deux filtres à houle Neyrpic destiné à purifier la houle et à absorber les réflexions produites par le batteur. Nous renvoyons pour la description et la théorie de ce «filtre » à l'article de M. Bresel paru dans la Houille Blanche (8) qui donne des photographies de ces deux appareils.
Par la suite, nous avons pu disposer d'un canal semblable au précédent. Sa longueur était toutefois un peu plus grande $(27 \mathrm{~m})$. Il comportail de plus une paroi vitrée: de ce fait, les mesures relatives au point de déferlement ont été plus commodes et plus précises.

Une seule pente a été essayée, de valeur $\frac{1}{10}$ (essai 7).

C'est sur ce canal qu'ont été pris les films dont il sera question plus loin.

\section{Mesures.}

a) Amplitude. - L'amplitude de la houle était mesurée au moyen d'appareils à pointe à œil cathodique d'un emploi courant au Laboratoire; ils étaient situés entre le dernier filtre et le début du plan incliné, aux nouds et ventres du clapotis partiel produit par la réffexion; de cette facon, en faisant la moyenne des lectures, on obtenait l'amplitude de la houle incidente.

b) Période. - La période était mesurée au moyen d'un compte-secondes sur 100 périodes du batteur; elle a été maintenue constante et igale à une seconde pendant tous les essais, sauf pour l'un des films. Notre but était en effet d'opérer sur des variables sans dimensions et nous avons choisi une période qui s'adaptait bien aux caractéristiques du canal.

c) Point de déferlement et caractéristiques de déferlement. - Nous parlerons plus loin des « critères » de déferlement, c'est-à-dire de ce qu'on doit entendre par « point de déferlement $\gg$.

En ce qui concerne l'estimation de ce point, nous avons opéré de la facon suivante pour le canal ne comportant pas de paroi vitrée : l'observateur se plaçait à une hauteur convenable de façon à pouvoir suivre la díformation de la crête sur une paroi du canal; un aide déplacait un repire qui servait à marguer le point de déferlement. Cette facon d'opérer est assez incommode et il est bien entendu préférable de disposer dune paroi vitrée. Cependant, avec un pen d'habitude, on arrive à des résultats assez précis; ainsi, ceux que l'on obtient de cette façon sur le premier canal avec la pente $\frac{1}{11}$ se raccordent bien avec ceux que donne la pente $\frac{1}{10}$ sur le deuxième canal. 

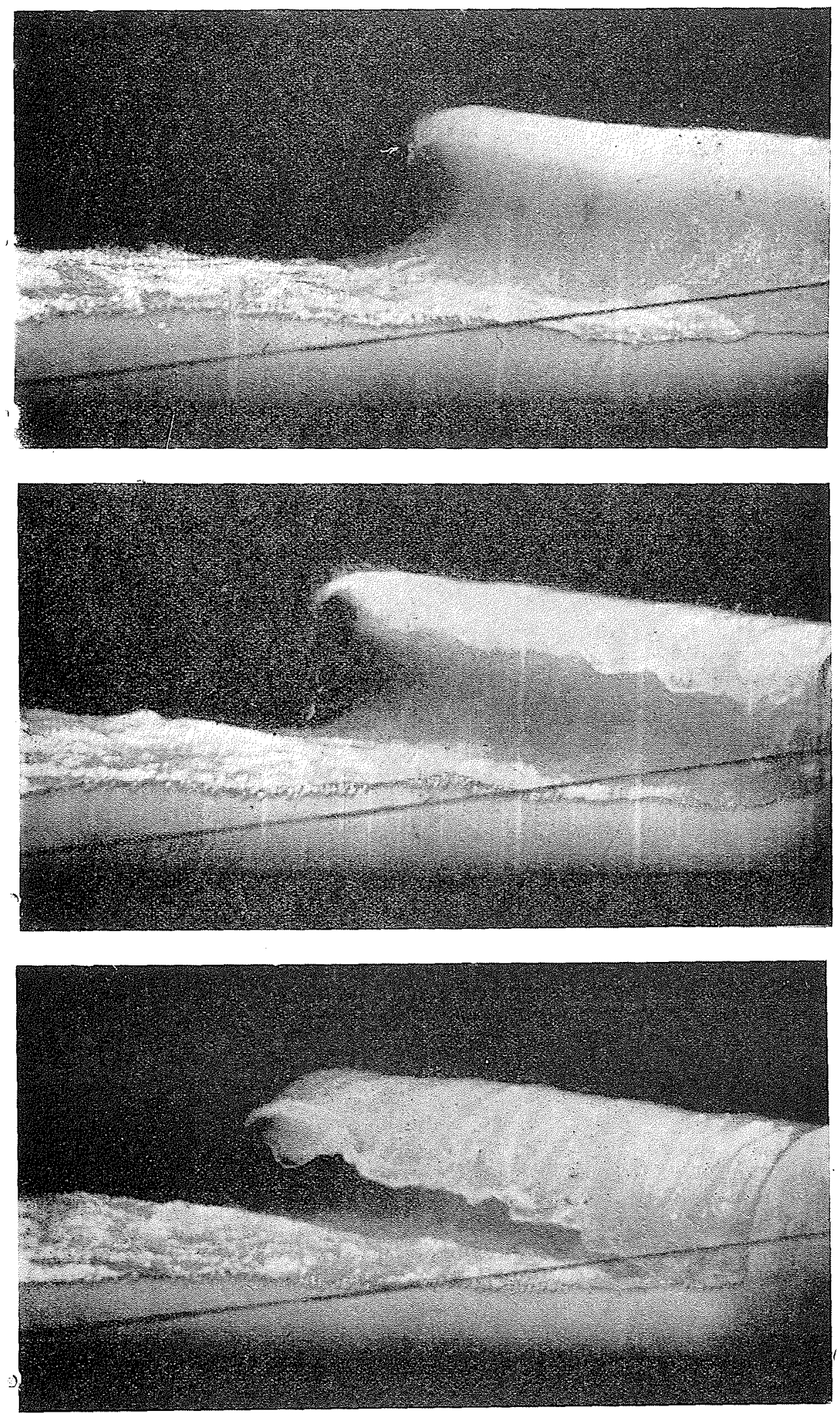

Fig. 2. - DÉfERlement eN VOLUTE MONTRANT LE « PLONGEON 》 DE LA GRÊTE 
La profondeur de l'eau au repos était mesurée au point de déferlement au moyen d'une pointe simple: on faisait deux lectures, une pour le niveau de l'eau au repos, l'autre pour le fond; la différence donnait la profondeur de déferlement.

Pour l'essai 7, fait sur le deuxième canal, l'observation et les mesures claient facilitées par la paroi vitrée. Pour les mesures, on avait disposé sur la vitre un « cache » en tóle peint en noir, comportant des graduations horizontales et verticales. La pente avait été étalonnée au préalable, c'est-a-dire qu'on avait tracé la courbe donnant les profondeurs en fonction des divisions de la graduation horizontale. Il suffisait pour repérer la position du point de déferlement de lire la division horizontale correspondante; on en déduisait la profondeur de déferlement au moyen de la courbe d'étalonnage. Cette dernière était d'ailleurs très sensiblement une droite.

3. Prises de vues cinématographioues. - C'est sur cette dernière installation qu'ont été faites des prises de vules cinématographiques s'étendant sur quelques périodes de houle $\left({ }^{*}\right)$. Le nombre de vues par seconde était voisin de 140 et a été contrôlé comme nous le verrons plus loin.

Nous avons ainsi étudié :

une houle de 1 seconde de période et de $4,7 \mathrm{~cm}$ d'amplitude en profondeur de $59 \mathrm{~cm}$. Nous la désignerons sous le nom de houle I;

une houle de 1 seconde de période et $8,8 \mathrm{~cm}$ d'amplitude pour la même profondeur. Nous la désignerons sous le nom de houle II;

une houle de 2,1 seconde de période. Ce dernier film était pris de trois-quarts alors que les deux précédents l'étaient sensiblement de profil.

\section{II. - Description du phénomène.}

\section{DIFFÉRENTES SORTES DE DÉFERLFMENT.}

Nos essais nous ont permis de constater qu'il existait plusieurs sortes de déferlements, ce qui était déjà connu d'après des expériences en laboratoire et des observations à la mer.

Soit d'abord une houle de cambrure modérée (3\% pour fixer les idées) arrivant sur un fond constitué par le plan incliné de pente $\frac{1}{5,7}$.

( ) Le nombre d'images pax secondes étant élevé nous étions en effet limités par la longuevr de film contenue dans un demi-chargeur $(60 \mathrm{~m})$; si l'on tient compte de la longueur nécessaire pour la mise en vitesse on conçoit que le nombre de périodes de houle pour lequel la vitesse de prise de vue est rigoureusement constante, soit peu élevé.
Le profil de la houle devient fortement dissymétrique, la pente du front étant plus raide que celle du versant arrière; à un moment donné, il se forme à la crête une singularité qu'on peut considérer comme un « point anguleux », sur laquelle nous reviendrons; ensuite, la dissymétrie du profil s'accentuant et la crête anguleuse s'inclinant de plus en plus, il arrive un moment où le front de l'onde présente une tangente verticale; la crête anguleuse continuant à s'incliner, elle devient surplombante puis bascule, s'enroule en quelque sorte et rattrape la base du front au moyen d'un jet ayant la forme d'une volute (fig. 2). Cette volute s'étale sur un petit espace en formant un rouleau : l'air emprisonné mêlé à l'eau donne de l'écume en se dégageant. Cet étalement, du fait de la valeur élevée de la pente, se produit en un point momentanément découvert par l'eau. Sur notre modèle comportant un fond en bois, il se produisait un clacquement sec. Un « jet de rive» résultat de l'étalement de la volute remonte la pente, animé d'une vitesse assez grande et redescend en produisant un « flot de retour » également assez rapide. Ce dernier rencontre la vague suivante sur le point de déferler et a une influence importante sur son déferlement.

Considérons maintenant une houle de même cambrure sur une pente faible, $\frac{1}{25}$ par exemple.

La houle commence par se déformer; le profil devient dissymétrique, mais beaucoun moins cue dans le cas précédent et la dissymétrie est d'autant moins accusée aue la pente de la plage est plus douce et la cambrure plus faible. Puis la crête présente un « point anguleux » beaucoup plus marqué que celui que nous avons envisagé précédemment et la pente du front s'accentue, mais sans jamais présenter de tangente verticale. A un certain moment, le déferlement s'amorce: des particules d'eau commencent à s'ébouler sur le front à une vitesse d'abord faible, mais qui s'accentue de plus en nlus, puis la masse s'écroule en formant un rouleau oui continue à avancer vers la rive en s'étalant; le « jet de rive » ainsi produit est plutôt une nappe d'eau mince poussée en quelque sorte nar les rouleaux suivants, cu'un jet véritable; le flot de retour a également une vitesse faible.

Ces deux modes de déferlement sont à rapprocher de ce que les Américains appellent les «plunging» et «spilling breakers » et qui ont d'ailleurs été observés dans la nature. Il est difficile d'en donner une description complète: nous renverrons le lecteur aux documents (9) (10) (11). Nous dirons cependant que dans le premier type, il se produit un «plongeon » de la crête, tandis que, dans le deuxième cas, le phénomène, tout à 
fait progressif, s'apparente au déferlement partiel en eau profonde.

Les déferlements que nous avons observés sont toutefois un peu différents. Le premier mode de déferlement que nous avons envisagé, et que nous appellerons « déferlement en volute » à la suite de M. Larras (4) et de M. Biestel (12) est bien un «plunging breaking», mais le deuxième mode que M. Bieset appelle « déferlement en deux temps » comporte également un plongeon.

Nous n'avons pas observé le « spilling breaking $\gg$ complet où la destruction d'énergie est entièrement progressive. Cela vient sans doute de ce que les plages sur lesquelles nous avons opéré n'avaient pas une pente suffisamment faible. Mais il est peut-être possible, comme le fait remarquer M. O’BRIEN (11) que le «plunging breaking $\gg$ se produise toujours ou presque en Laboratoire, mais qu'en mer, dans certains cas, il se produise un «spilling breaking », des causes accidentelles pouvant èmpêcher le « plunging » de se produire; ce pourrait être par exemple le vent, des irrégularités du fond, des courants, des ondulations parasites superposées à la houle, etc...; ces causes accidentelles ont d'autant plus de chance d'intervenir que le temps mis par la houle pour déferler est plus grand, c'est-à-dire que la pente est plus faible et que les houles ont des dimensions moins importantes.

En résumé, en ce qui concerne les modes de déferlement que nous avons observés, les différences sont les suivantes:

a) Dans le cas du « déferlement en deux temps $\gg$, il n'apparaît pas de tangente verticale sur le front de l'onde avant le commencement de l'éboulement des particules d'eau;

b) Toujours dans ce même cas, cet éboulement est progressif le long du front. Pour le «déferlement en volutes », il y a, au contraire, incurvation et plongeon brutal de la crête;

c) Dans le cas du « déferlement en volute», la volute s'étale sur une très courte distance en percutant le fond sur lequel elle produit des effets mécaniques intenses (formation de barres); les vitesses sont très grandes. Dans le cas du « déferlement en deux temps », le phénomène est progressif, surtout au début, et il l'est d'autant plus que les pentes sont plus douces; en particulier, l'étalement du rouleau n'est pas brutal et l'on voit se suivre sur la plage au même instant plusieurs rouleaux provenant de vagues successives. Les vitesses d'écoulement sont moins grandes que dans l'autre cas.

Cette différence explique que la pratique du "surf-riding » ne soit possible que sur des plages très plates; ce sport consiste à se laisser por- ter à la côte sur un bateau spécial en se maintenant au voisinage du sommet des rouleaux de déferlement;

d) Le jet de rive est beaucoup plus rapide que dans le cas du déferlement en volutes; il en est de même pour le flot de retour.

Les dessins de la figure 3 schématisent les différentes formes.

Il existe d'ailleurs tous les types intermédiai-
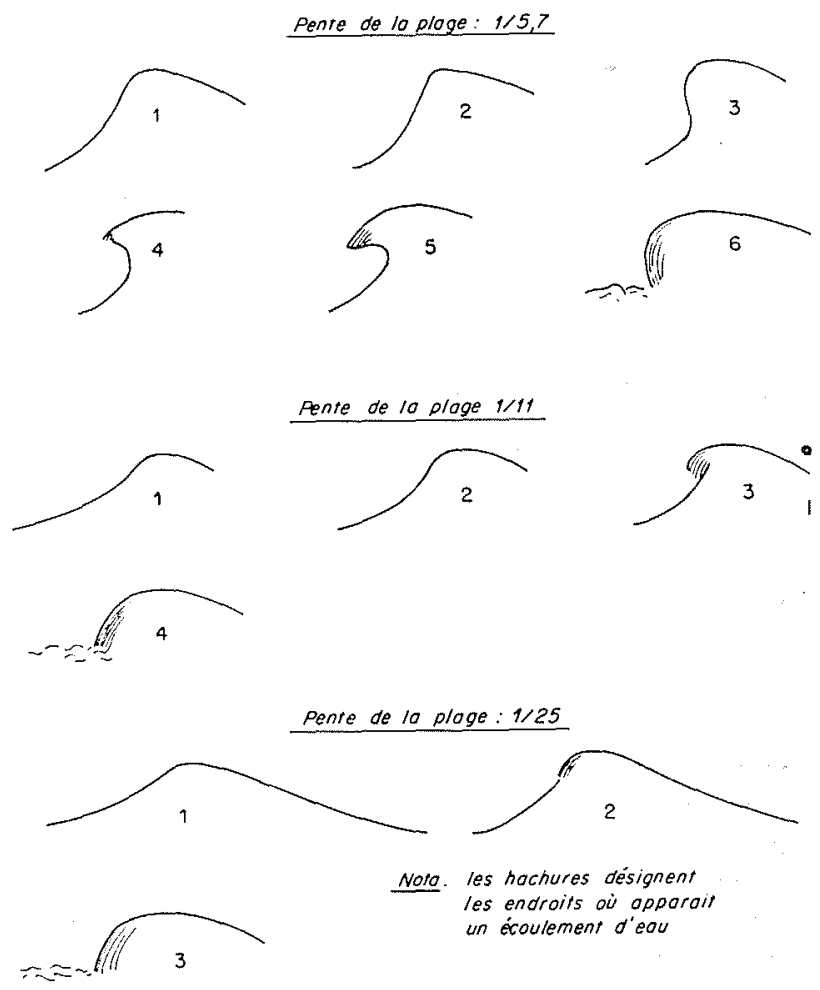

Fia. 3. - Schémas, montrant les différentes phases et formes du deferlement

res entre les deux types de déferlement décrits ci-dessus. Par exemple, les figures 4 et 5 indiquent les différentes phases du déferlement des houles $n^{\circ} I$ et II que nous avons cinématographiées et qui déferlaient sur une pente de valeur $\frac{1}{10}$, par conséquent comprise entre les deux cas extrêmes que nous avons envisagés. Sur ces photos, le front de l'onde ne présente jamais de tangente verticale; cependant, pour la houle I, en considérant le film complet, on voit une tangente verticale très fugitive (fig. 6) qu'on ne retrouve pas sur le film de la houle II. D'ailleurs, pour ces deux houles, on n'aperçoit sur la plage

(*) Sur les rivages sablonneux, la « barre 》 (bourrelet de sable suivi d'un creux) se produit au voisinage immédiat de ce point. 

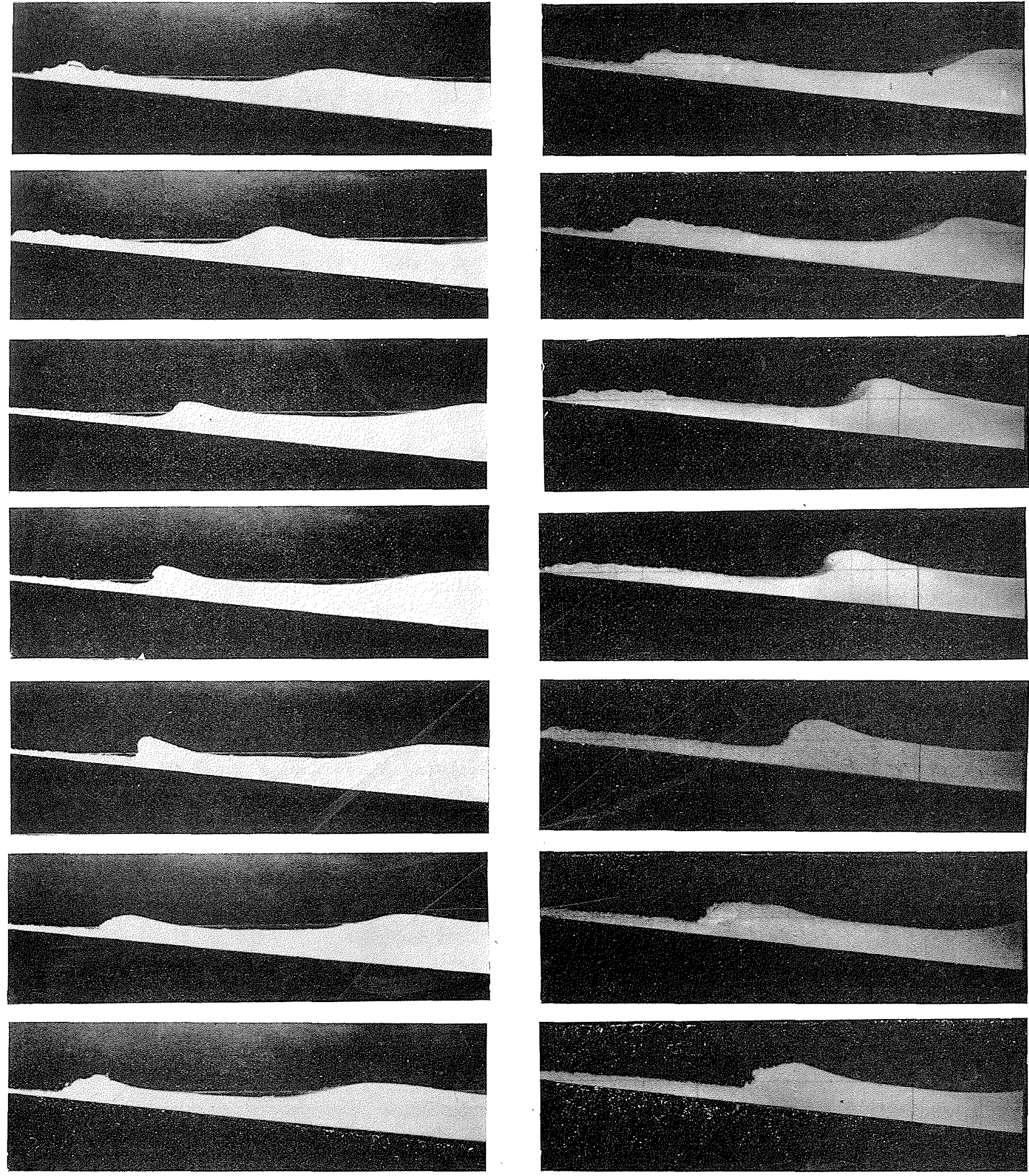

Fig, 4. - Hovle I : Phases sucessives du déferlement

Fig. 5. - Hovle II : Phases sucessives du deferlement 


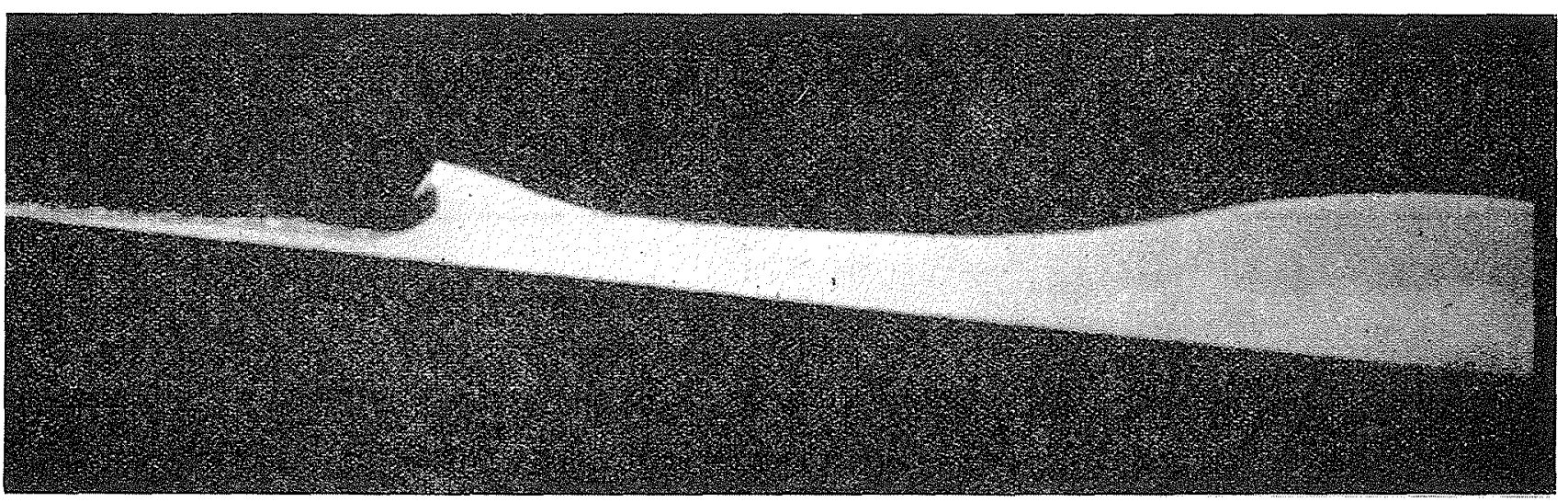

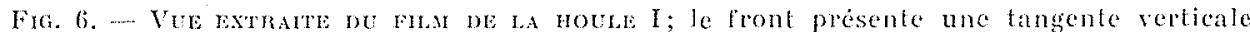

qu'un rouleau de déferlement et il existe un "plongeon» de la crête assez net ainsi qu'un jet de rive et un flot de retour assez importants.

\section{Fissai dinterpefitation.}

D’après M. Bieser (12), il semblerait que le déferlement résulterait de la combinaison de deux effets :

a) un raidissement de la face avant de la vague dù à la pente du fond, raidissement d'autant plus important à profondeur égale que la pente est plus forte. Cet effet est encore mal connu, mais une explication intuitive en est donnée par M. Mrche (1);

b) la diminution de profondeur qui produit pratiquement une augmentation de la cambrure; la houle peut se rapprocher de sa forme limite; il se produit alors un « prédéferlement » ressemblant au déferlement partiel de la houle en eau profonde dont nous avons parlé plus haut.

Suivant la pente, l'un ou l'autre effet serait prépondérant. Nous y reviendrons dans le paragraphe suivant.

D'autres effets interviennent également.

Il existe, surtout dans le cas d'un « déferlement en volute $»$, une réflexion sur la pente avec formation d'un clapotis partiel. Ce dernier phénomène modifie l'amplitude de la houle incidente et intervient ainsi certainement sur le point de déferlement.

Enfin, le flot de retour, beaucoup plus important dans le cas d'une pente forte, produit un double effet sur le phénomène: d'une part, le courant de sens contraire à celui de la propagation de la houle, déplace vers le large le point de déferlement; d'autre part, la quantité d'eau ainsi rejetée engendre des ondes; ce phénomène, dans nos essais, était d'ailleurs inséparable de la réflexion partielle.

Quand la pente devient très forte $(1 / 1$ par exemple), le clapotis partiel est prépondérant et le phénomène de "brisure » observé n’a que lort peu de rapport avec le déferlement d'une houle sur pente peu inclinée.

Nous verrons plus loin (2" partie, III), que pour une pente de fond $\frac{1}{10}$, le coefficient de reflexion est de lordre de $16 \%$ pour une houle de cambrure au large $3 \%$. On voit également sur les figures 4 et 5 des ondes secondaires, mais il nous semble cependant difficile de les attribuer à la réflexion ou au flot de retour, car on ne peut déceler de propagation nette. Nous y reviendrons.

\section{NATURE DE LA SINGULARTÉ A LA CRETE.}

Nous avons dit qu'au début du déferlement il apparaissait dans certaines conditions une singularité à la crête de la vague. En fait, sa nature varie beaucoup avec la pente.

Pour une pente très forte (à partir de $\frac{1}{3}$ à $\frac{1}{2}$ pour fixer les idées), le versant arrière de la vague reste constamment convexe vers le haut et il n'apparaît rien qui ressemble à un point anguleux.

Cette convexité du versant arrière persiste jusqu'à des pentes de la plage assez faibles. Ce n'est qu'à partir d'une pente $\frac{1}{15}$ ou $\frac{1}{25}$ que le front arrière présente une concavité franche vers le haut, qui rapproche ce déferlement de la forme limite de la houle en eau profonde. Sur la figure 7 (photographie extraite d'une autre série d'essais), on voit ainsi une houle de cambrure au large $2 \%$ sur le point de déferler par fond de pente $1 / 50$.

A mesure que la pente diminue, la crête déferlante présente une région de courbure de plus en plus faible et il semble que c'est à ce moment 
là qu'on peut parler de point anguleux. Pour la pente $\frac{1}{10}$, on observe les faits suivants (fig. 4 et 5) : il se forme d'abord un méplat situé au voisinage, mais sur l'avant de la crête; ce méplat est peut-être dù à la réflexion, mais il s'est produit de facon à peu près identique pour les deux

\section{Influence de da CAMBRURE.}

Nous n'avons fait intervenir jusqu'à présent que la pente de la plage; la cambrure de la houle au large entre aussi en ligne de compte.

Imposons-nous comme caractéristique conventionnelle pour le «déferlement en volute»

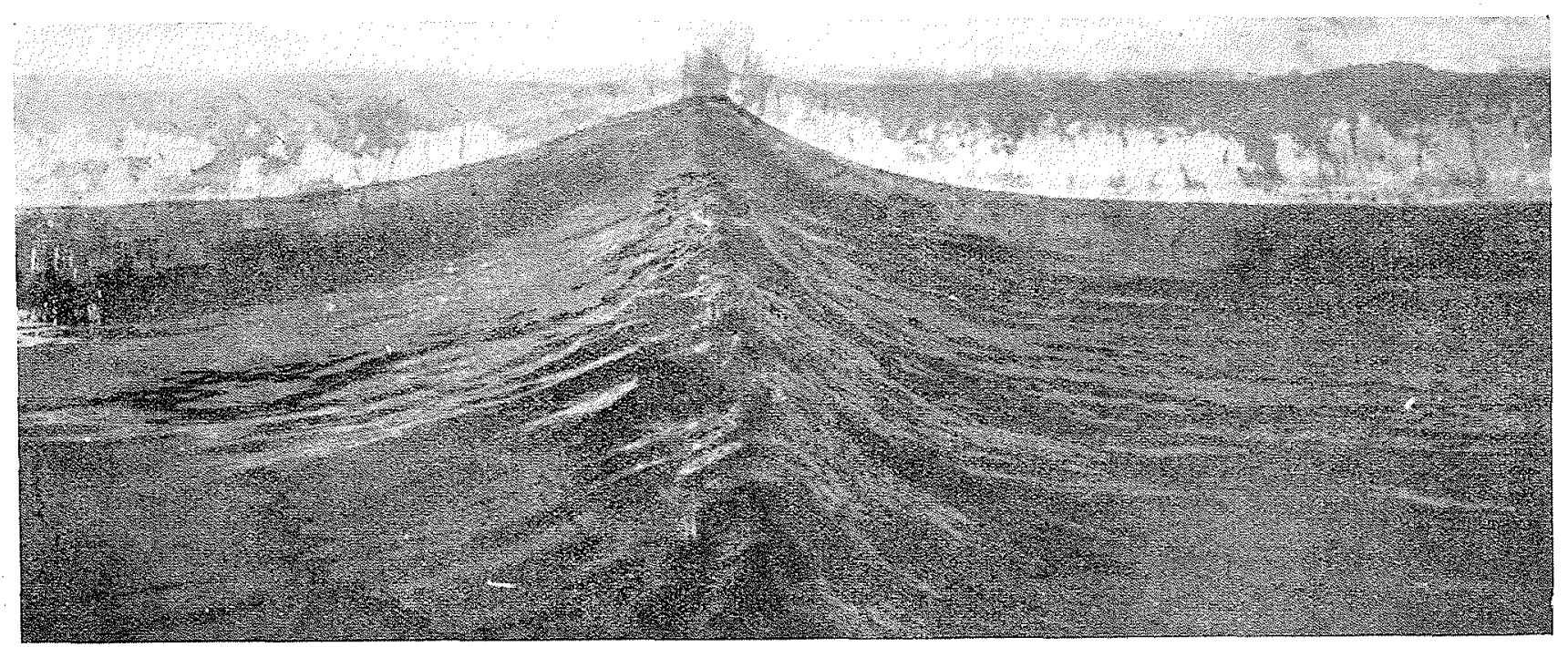

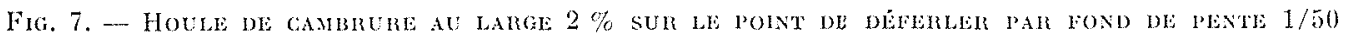

houles que nous avons cinématographiées; il se déplace jusqu'à devenir horizontal; c'est à ce moment-là que le rayon de la courbure de la crête est le plus faible et qu'on peut parler de point anguleux; la pente du front devient alors maximum et l'éboulement des particules commence. Mais l'aspect du phénomène est bien éloigné de celui de la houle limite en profondeur constante.

Le point anguleux de la crête n'est jamais bien net dans les expériences sur modele; cela ne signifie pas obligatoirement que la singularité de la crête ne puisse être représentée par un point anguleux; la capillarité intervient en effet et s'oppose à la formation de tout angle vif. De plus, ce qui a été photographié et ce que nous avons observé était la déformation d'un profil de houle sur une paroi; le frottement sur la paroi peut avancer l'éboulement des particules à ce moment où la crête est très instable. C'est d'ailleurs ce que semble montrer le film de la houle II (fig. 5) qui n'a pas été exactement pris de profil.

Quoi qu'il en soit, si l'on adopte l'explication intuitive du déferlement donnée en 2 , on doit admettre qu'à moins d'angle du fond très faible, l'effet prépondérant est celui causé par la pente. la particularité pour le front de posséder une langente verticale; si nous portons la pente de la plage $p$ en abscisses, la cambrure au large de la houle $\psi_{0}$ en ordonnées, les essais semblent bien montrer que la courbe représentant la frontière entre les deux régions aura l'allure représentée par la figure 8. Nous avons vu par exemple que pour la pente $\frac{1}{10}$, le front d'une houle correspondant à un petit $\gamma_{0}$ semble présenter

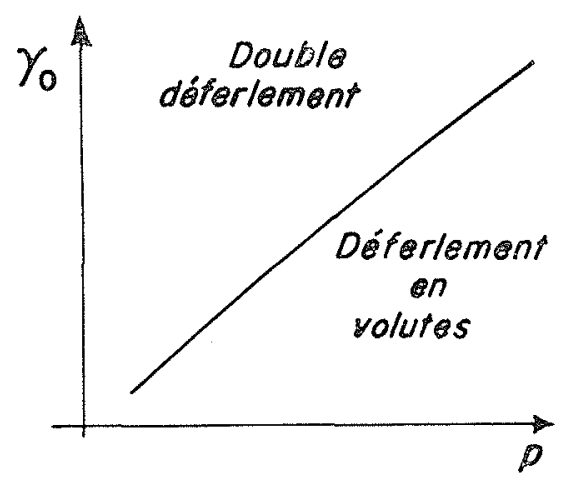

Fra. 8

une tangente verticale alors que pour un $\%_{0}$ plus élevé, ceci ne se produit pas.

Les houles plates (correspondant à un $\gamma_{0}$ fai- 
ble) déferlent en volute pour des pentes plus douces que les houles cambrées. Nous n'avons malheureusement pas pu faire de mesures; seule la cinématographie de la houle permettrait une étude complète. Le fait précédent est d'ailleurs conforme à l'explication donnée en 2; quand la cambruxe au large est forte, la houle peut se rapprocher plus vite de sa forme limite et a par conséquent tendance à déferler partiellement. L'inverse se produit pour les houles plates.

Cette propriété ne s'applique vraisemblablement qu'aux houles pures produites en laboratoire. En mer, le phénomène peut ètre inversé; nous avons signalé en effet en II, $\S 1$, les causes qui peuvent amorcer un déferlement partiel; ces causes ont en mer une importance relative d'autant plus grande que les houles sont à une «échelle» plus petite.

\section{III. - Point de déferlement. Critère de déferlement}

Il nous faut maintenant convenir du point où seront mesurées les caractéristiques de la houle déferlante; nous le ferons à la lumière de ce cqui précède.

On serait tenté d'appeler « point de déferlement $\gg$ le point où la houle présente un point anguleux. Mais ainsi que nous l'avons vu, il n'apparaît jamais en pratique de facon nette et dans certains cas il n'existe certainement pas.

On peut aussi choisir le point où le front présente pour la première fois une tangente verticale. Il est assez facile à localiser à l'wil ou au moyen d'un film, mais ce critère ne peut s'appliquer à tous les types de déferlement. Nous l'avons employé systématiquement pour l'essai 1 et pour quelques points des essais 2 et 7 .

Pour les autres pentes, deux critères viennent à l'esprit. On peut choisir le point où des particules d'eau commencent à s'ébouler le long du front, point qui, dans le temps, doit suivre de peu le point où la crête devient anguleuse. On peut également utiliser le point où la pente du front est maximum avant l'écroulement de la crête, mais alors que l'écoulement de l'eau a déjà commencé; il suit de peu ce dernier point et précède le point de formation du rouleau.
Ces deux points sont assez difficiles à localiser à l'œil, mais sont faciles à repérer sur un film. Le premier présente l'inconvénient de ne pas se « raccorder » au point de vue théorique au critère de la tangente verticale.

Au cours de nos derniers essais, nous avons pensé qu'on pourrait utiliser le point situé à l'extrême avant du rouleau qui se forme après l'écroulement plus ou moins brusque de la crête. Nous avons utilisé ce critère dans notre dernier essai (essai 7); le point en question est très facile à observer à l'œil et les points expérimentaux se placent tròs bien sur une courbe (fig. 9).

Il vaudrait mieux d'ailleurs définir un critère d'apres des considérations théoriques, en admettant qu'il soit possible d'élaborer une théorie qui s'applique à tous les modes de déferlement. Dans l'état actuel des choses, un critère de déferlement entièrement satisfaisant semble difficile à trouver : le meilleur pour les essais que nous avons effectués aurait sans doute été le point situé à l'extrême avant du rouleau à sa formation.

\section{IV. - Longueur de déferlement.}

La notion de profondeur de déferlement est bien insuffisante pour caractériser quantitativement le phénomène. Il nous semble qu'il serait nécessaire d'introduire la notion de « longueur de déferlement $\gg$, distance entre le début et la fin du déferlement; pour le début, on pourrait prendre le point où les particules d'eau commencent à s'ébouler sur la crête ou celui où le front de la vague présente une tangente verticale; la fin du déferlement serait le point avant du rouleau dont nous avons parté en III, point facile à localiser.

D'après ce qui précède, la longueur de déferlement dépend, non seulement de la pente, et des caractéristiques de la houle, mais également de la forme des fonds, du vent et des courants et aussi de la «pureté » de la houle. Pour une côte donnée, on pourrait ainsi définir une « zone dangereuse au déferlement », résultant de l'étude statistique des diverses houles susceptibles de se produire sur celte côte; cette considération serait importante par exemple pour la construction d'un warf sur une côte à barre. 

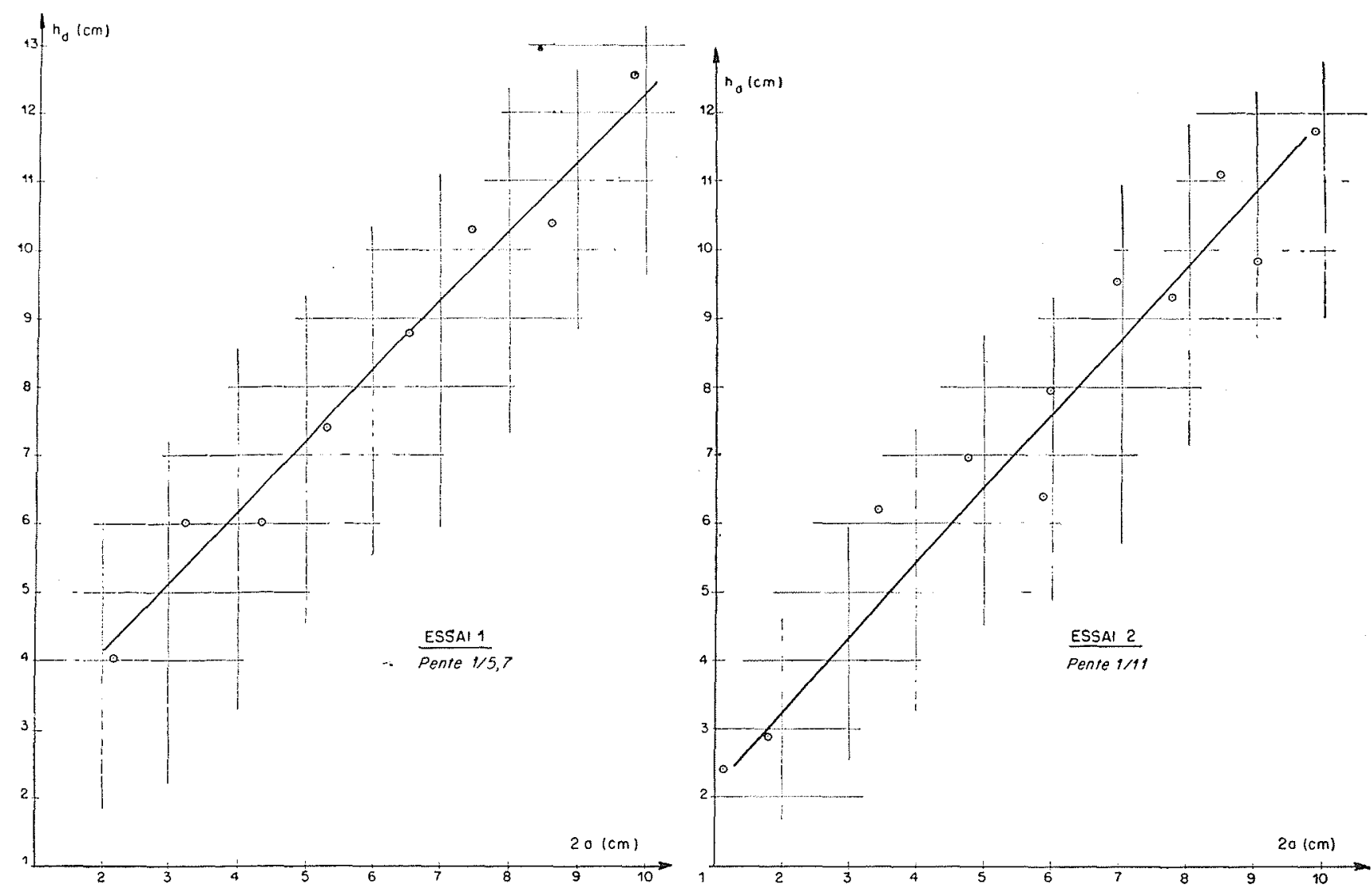

ESSAI 2
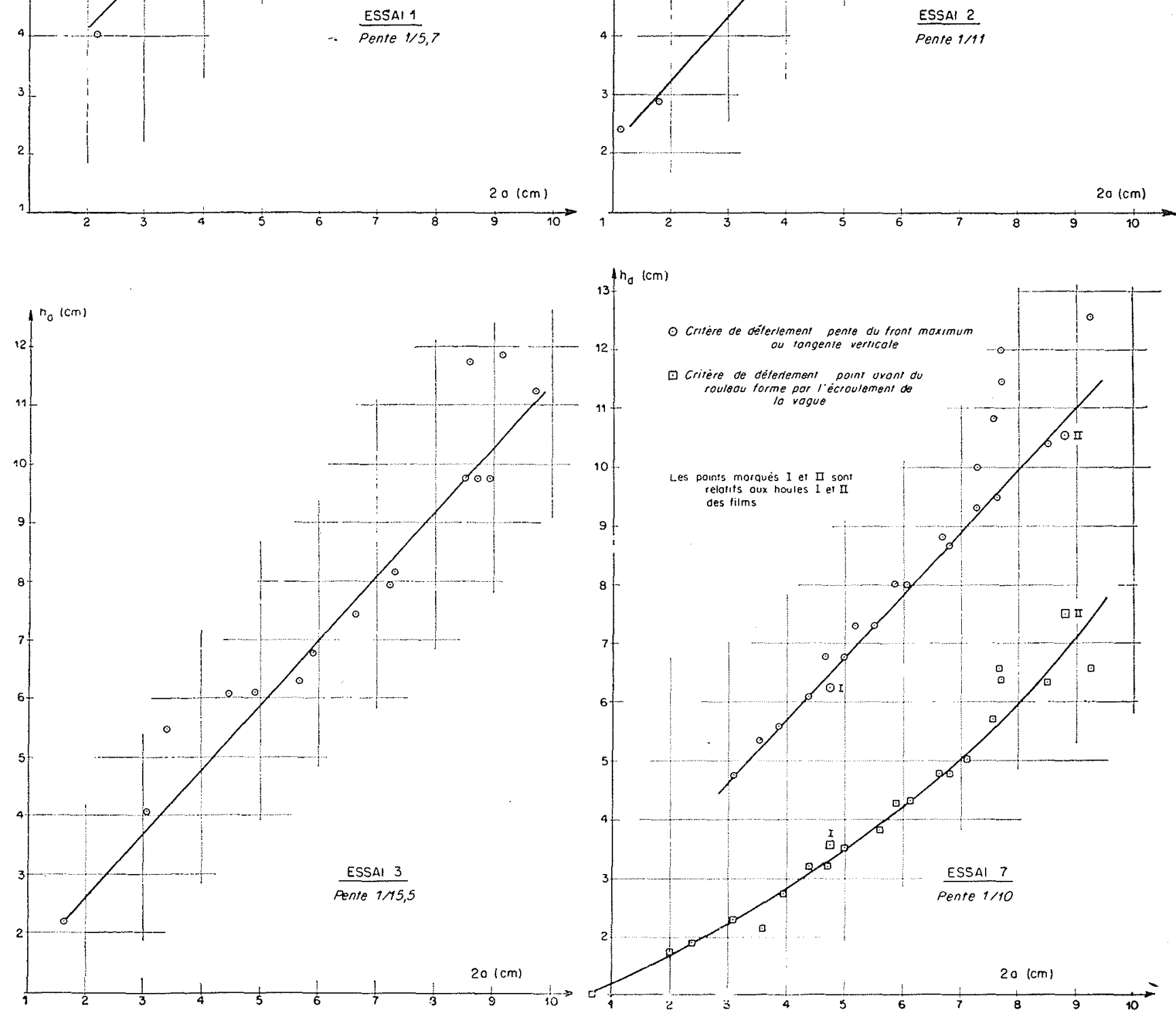

FIG. 9 


\section{DEUXIEME PARTIE}

\section{RESULTATS EXPERIMENTAUX ET INTERPRETATION}

Le nombre d'essais effectués (53 points expérimentaux) est faible si l'on tient compte de la complexité du phénomène; de plus, la dispersion des mesures est assez grande, sauf pour l'essai 7 et les résultats que l'on peut extraire des films, car, dans ces derniers cas, les conditions expérimentales étaient bien meilleures. Cependant, pour chaque essai, il est possible de tracer sans difficultés une courbe moyenne qui a une signification : la preuve en est que les courbes relatives aux essais 2 et 7 effectués respectivement avec des pentes égales à $\frac{1}{11}$ et $\frac{1}{10}$ sont très voisines et que leur écart est bien dans le sens que l'on pouvait prévoir.

Nous devons distinguer trois groupes d'essais : les essais 1,2,3,7 pour lesquels le plan incliné allait jusqu'au fond horizontal du canal;

les essais $4,5,6$ pour lesquels un raccordement

à allure grossièrement parabolique avait été ménagé entre le fond du canal et le plan incliné afin de ne pas donner à ce dernier une trop grande longueur; les films.

Nous avons tracé deux sortes de courbes:

1. Pour chaque essai, une courbe donnant la profondeur de déferlement $h_{\mathrm{d}}$ en fonction de l'amplitude mesurée en profondeur constante $2 a$.

2. Un graphique donnant $\frac{h_{\mathrm{a}}}{2 a_{0}}$ en fonction de $\gamma_{1}, 2 a_{0}$ et $\gamma_{0}$ étant respectivement l'amplitude et la cambrure en profondeur infinie; ces quantités ont été calculées au moyen d'une théorie approchée, mais vérifice par l'expérience, fondée sur la constance du débit d'énergie el appelée par M. Mrche « théorie énergétique ». Sur ce graphique sont portés les résullats des essais 1, 2, 3, 7. Les essais 4,5 et 6 ont été écartés car, comme nous le verrons, la présence d'un raccordement relativement brutal perturbe le phénomène el ne le rend pas comparable à ce que l'on observe dans les autres essais.

Dans ce qui suit, nous passerons d'abord en revue chaque groupe d'essais en examinant linfluence de diférents facteurs sur le déferlement et en étudiant l'évolution de certaines caractéristiques de la houle au voisinage du point de déferlement. Ensuite, traçant un graphique don- nant $\frac{h_{\mathrm{i}}}{2 a_{0}}$ en fonction de $\gamma_{0}$, nous rassemblerons les résultats sous une forme sans dimensions, ce qui nous permettra certaines comparaisons.

\section{I. - Groupe 1 (fig. 9). Influence de la pente.}

La pente semble avoir une influence sur la position du point de déferlement; plus la pente est forte, plus la profondeur de déferlement est grande pour une amplitude et une longueur d'onde données.

C'est ce que montre la courbe de la figure 10.

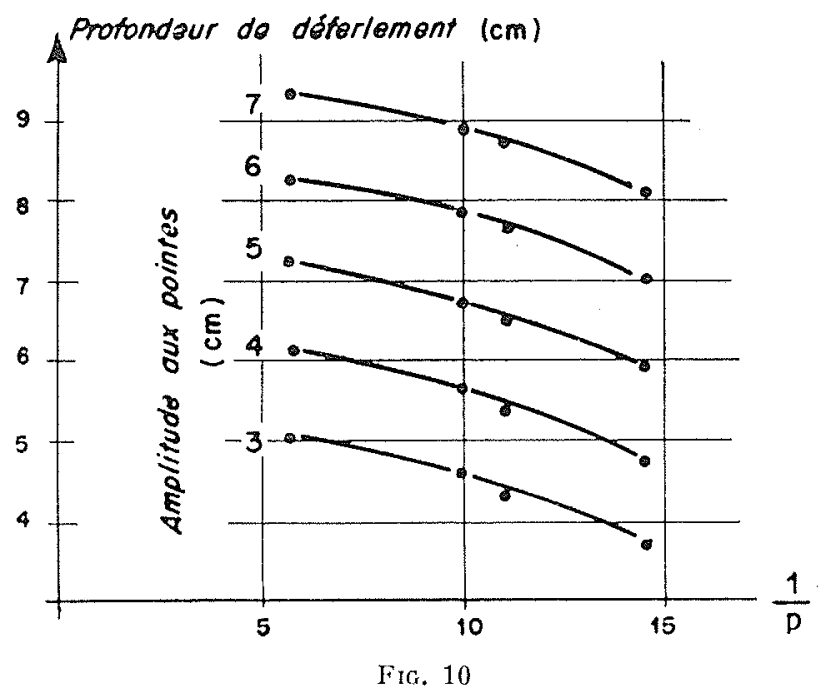

En toute rigueur, on n'aurait dû comparer que des résultats obtenus avec le même critère de déferlement; la courbe relative à l'essai 1 a en effet ćté tracée en adoptant comme critère celui de la tangente verticale, l'essai 2 en partie avec ce dernier critère, en partie avec celui de la tangente maximum, l'essai 3 avec le critère de la tangente maximum. Cependant, nous avons cru bon de faire intervenir l'essai 1 car l'essai 2 a montré que les deux critères se « raccordaient»assez bien et le critère de la tangente verticale ne peut plus s'appliquer à l'essai 3 .

L'influence de la pente sur le profil de la houle 

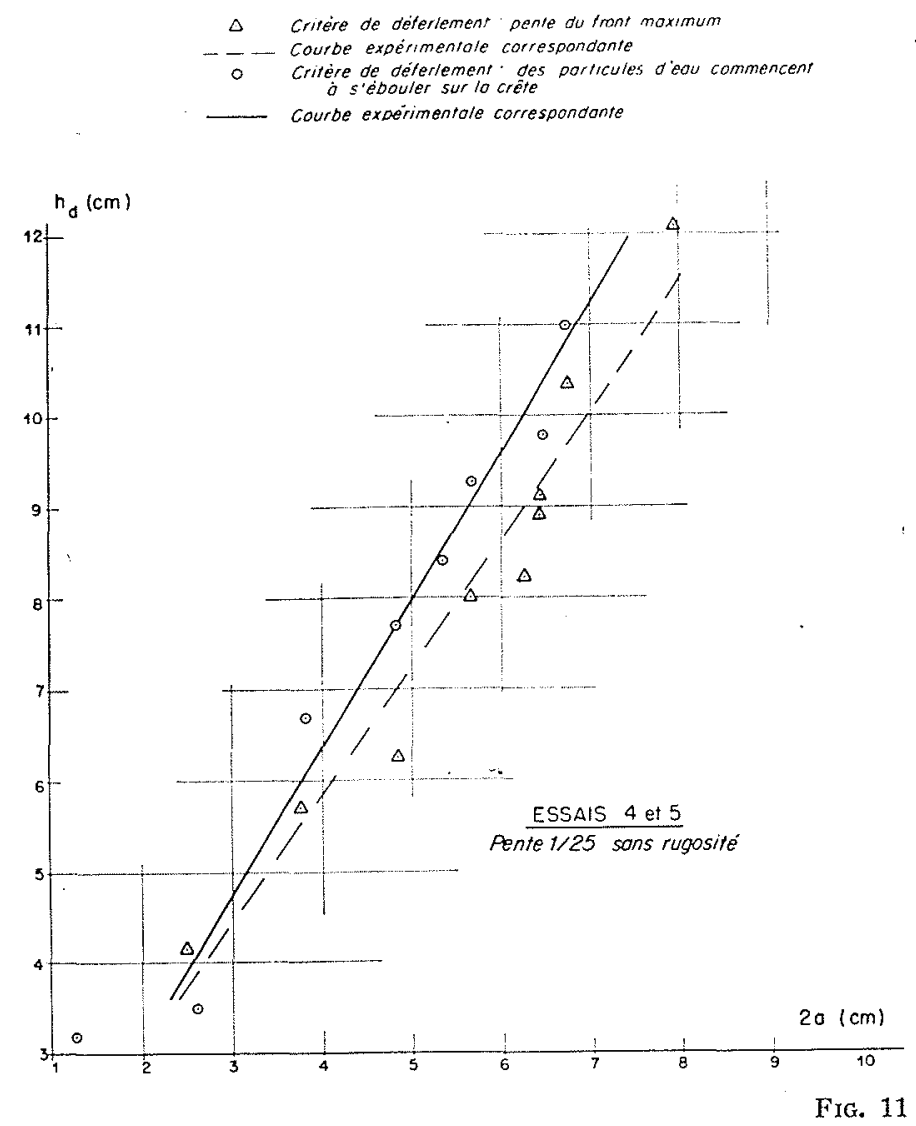

et le mode de déferlement a été examiné dans la première partie.

\section{II. - Groupe 2 (fig. 11).}

L'essai $n^{\circ} 4$ était fait avec une pente de $\frac{1}{25}$; le fond, ainsi que nous lavons indiqué, était constitué par un plan incliné d'une longueur de 8 mètres (fig. 1).

Les essais ont été faits en prenant successivement deux critères de déferlement : pente maximum du front et éboulement des particules d'eau sur le front; les courbes correspondantes sont décalées l'une par rapport à l'autre dans un sens qui pouvait être prévu. Toutes deux montrent que la houle déferle pour une profondeur nettement supérieure à celle des essais précédents (fig. 11).

Il ne semble pas qu'on puisse en tirer une conclusion relativement à la variation du point de déferlement avec la pente. En effet, il est bien connu qu'une discontinuité brusque du fond fait déferler la houle, même si la profondeur est supérieure à la profondeur de déferlement en fond lentement variable. Par analogie, on peut

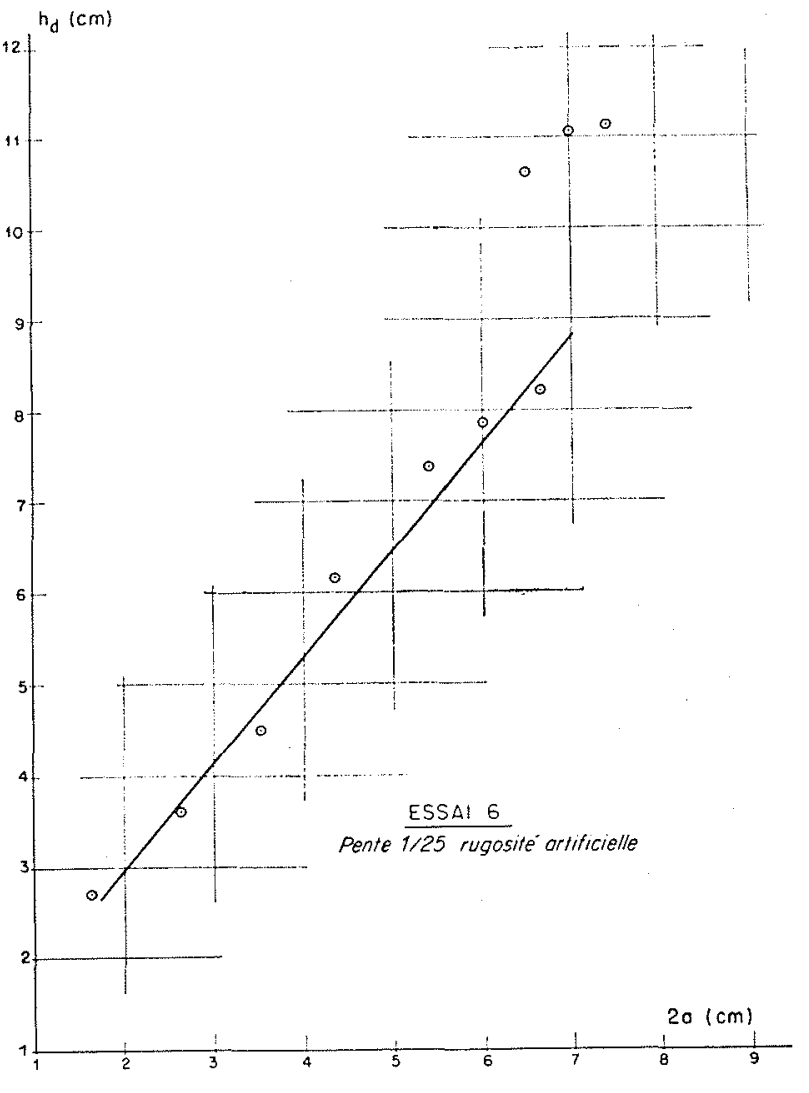

penser qu'une variation relativement brusque du fond perturbe le phénomène suffisamment pour augmenter la profondeur de déferlement.

Dans l'essai 6, le fond a été recouvert de métal déployé afin d'en augmenter la rugosité. On constate alors que la profondeur de déferlement est plus faible que lors de l'essai précédent. Deux explications viennent à l'esprit : le frottement sur le fond dissipant de l'énergie, l'amplitude de la houle diminue et par conséquent le déferlement se produit pour une profondeur plus faible comme le montrent MM. Putnam et Johnson (13); on peut penser également que le flot de retour, freiné par la rugosité du fond, a une influence plus faible sur le déferlement. Il nous a semblé cependant que pour une pente de $\frac{1}{25}$, la vitesse du flot de retour est faible et que le premier effet doit être le plus important. Mais l'importance des deux effets pourrait être inversée dans le cas d'une pente forte.

Nous n'avons pu faire d'autres mesures pour étudier l'influence de la rugosité sur le point $\mathrm{d} r$ déferlement et préciser l'importance relative des divers facteurs. Pour une pente de $\frac{1}{25}$, la rugosité semble donc déplacer vers le rivage le point 

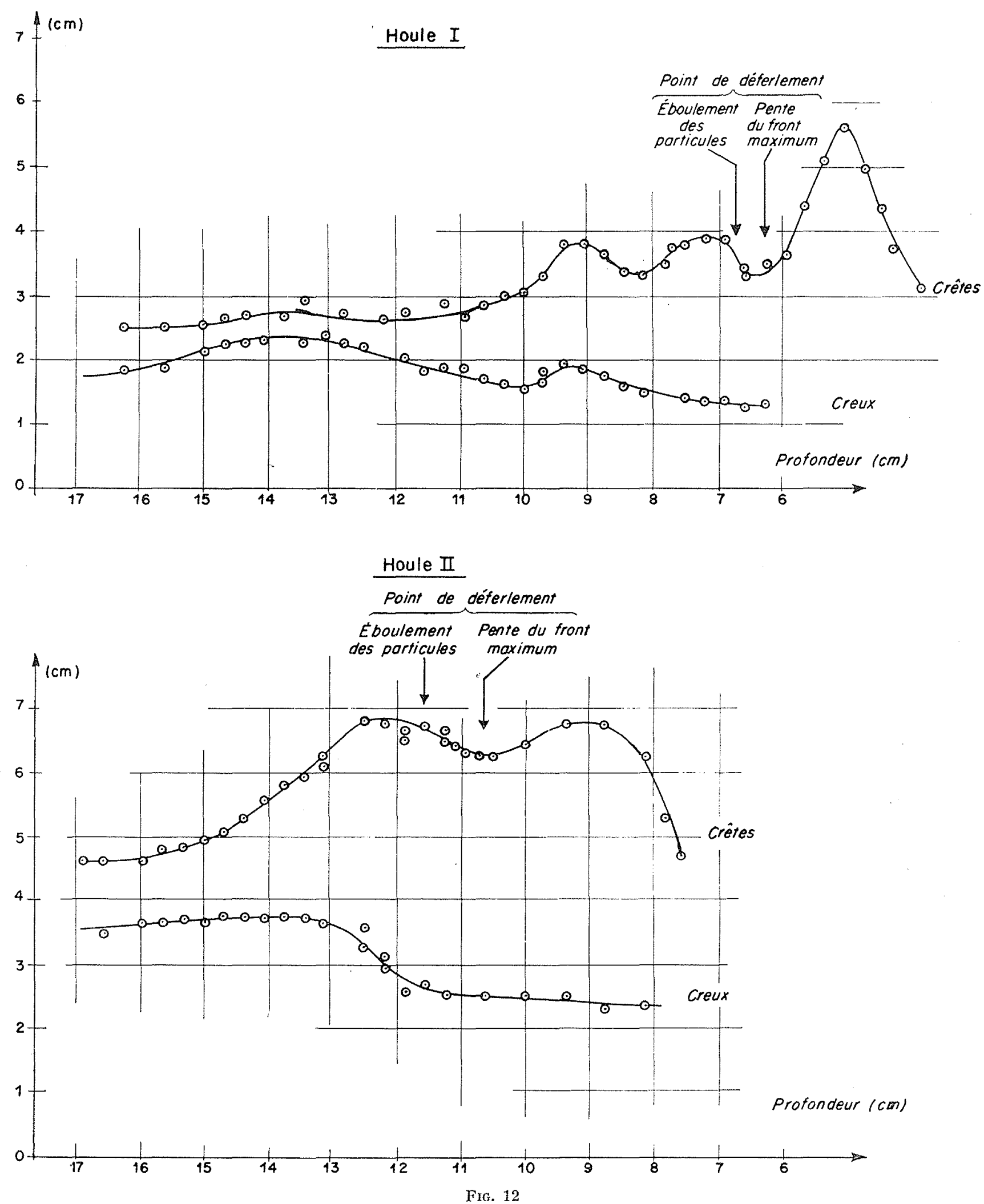

de déferlement, mais rien ne prouve encore qu'il en soit de même pour les fortes pentes.

Une conclusion qui semble se dégager de ces deux groupes d'essais et que font apparaitre les graphiques est celle de l'instabilité des houles d'amplitude supérieure à $8,5 \mathrm{~cm}$ correspondant 
à une cambrure de $6 \%$. Les points expérimentaux sont alors très dispersés. Ceei est dû certainement pour une part aux difficultés d'observation : le point à tangente verticale ou maximum et le point d'éboulement des particules sont difficiles à localiser exactement. Mais la même dispersion apparaît pour le critère du rouleau pourtant facile à localiser. Il semble donc bien que les houles de cambrures supérieures à une certaine limite sont instables et qu'une cause fortuite, une ondulation parasite par exemple, peut les faire déferler.

\section{III. - Groupe 3. - Films.}

Eyolution des CARACTÉristigues DE LA HOULE AU VOISINAGE DU POINT DE DEFERLEMENT

Les films ont été dépouillés au moyen d'un appareil servant à la lecture des micro-films. Un quadrillage était placé sur l'écran de l'appareil; il était ainsi possible de mesurer des longueurs; comme le «eache » placé sur la glace du canal portait deux échelles graduées, l'une horizontale, l'autre verticale, on pouvait ainsi en déduire les longueurs réelles.

Les points étudiés ont été les suivants : pente maximum du front et éboulenent des parlicules d'eau; elle a été trouvée égale à $7 \%$ environ pour la houle I et $9 \%$ pour la houle II; c'est l'ordre de grandeur qu'avaient fourni les essais 4 et 5 où les deux critères avaient été employés.

b) Hauteur des crêtes et des creux au-dessus et au-dessous du niveau de lean au repos. Variation de l'amplitude.-- Réflexion.

Faisant choix d'un point de profondeur donnée, on peut mesurer sur le film les cotes de la crête et du creux passant en ce point.

Nous obtenons ainsi les courbes de la figure 12 pour les houles I et II.

Ces courbes nous permettent d'abord de suivre la variation de l'amplitude avec la profondeur; nous définissons en effet l'amplitude en un point comme la différence des cotes de la crête et du creux passant en ce point.

Nous pouvons ainsi tracer les courbes de la figure 13.

Pour la houle I, cette courbe présente une forme ondulée. Nous avons tracé sur le mème graphique la courbe de la variation de l'amplitude donnée par la théorie énergétique; la courbe expérimentale serpente autour de la courbe théorique.

Les points étant asez bien groupés, on peut
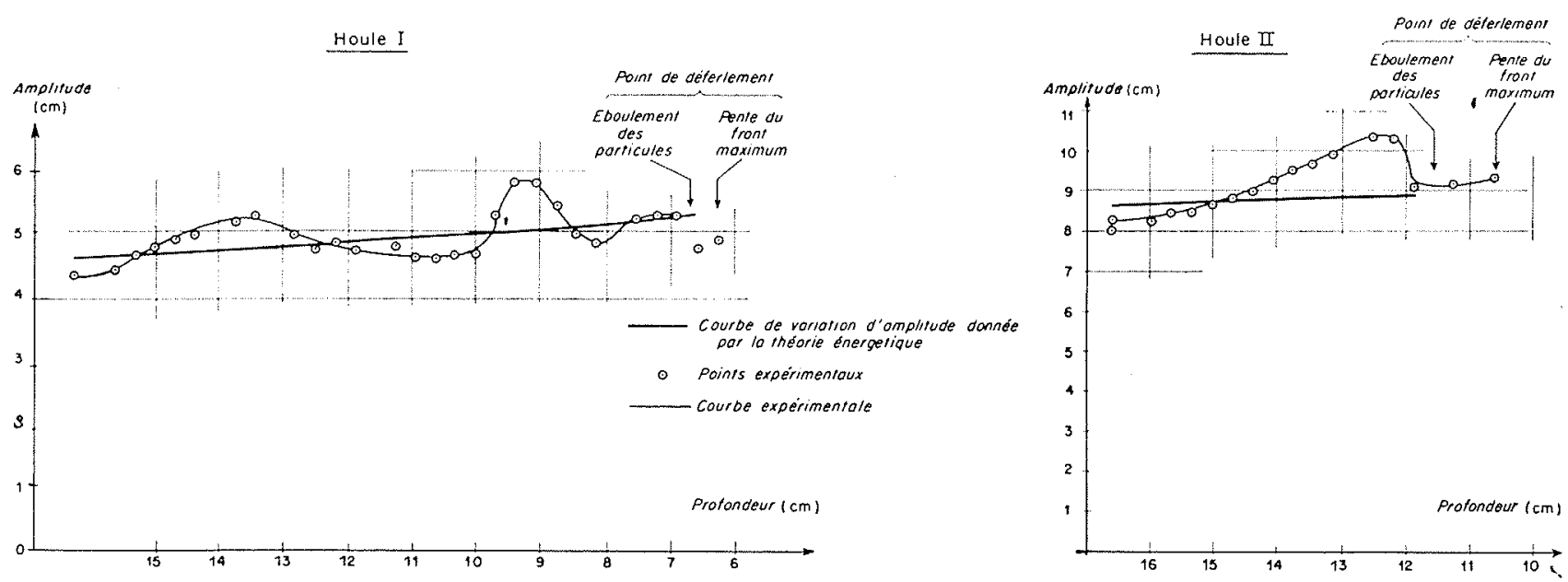

Fig. 13

\section{a) Point de deferlement.}

Il est très facile de localiser sur le film la position du point de déferlement. Ceux relatifs aux houles I et II ont été ainsi portés sur la courbe relative à la pente $\frac{1}{10}$ (fig. 9).

Il est également possible de se rendre compte de la différence entre les profondeurs de déferlement correspondant aux deux critères suivants : penser que ces ondulations de la courbe - du moins celles situées en profondeur relativement grande - sont l'indice d'une réflexion produite par la pente; devant la plage, existe de ce fait non pas une houle, mais un clapotis partiel que l'on peut considérer en première approximation comme la superposition de la houle incidente et de la houle réfléchie. L'amplitude varie donc lorsqu'on passe d'un point à un autre, mais 
en un point donnc, elle est constante dans to temps puisque le phénomène est permanent. En profondear constante, la distance entre deux maxima ou deux minima d'amplitude serait égale à une demi-longueur d'onde. Si sur la courbe de la figure on mesure la distance comprise entre les minima d'amplitude de profondeurs respectives égales à $16,2 \mathrm{~cm}$ et $10,6 \mathrm{~cm}$, on la trouve égale à $56 \mathrm{~cm}$; or, la demi-longueur d'onde pour la profondeur moyenne de $13,4 \mathrm{~cm}$ supposée constante serait de $51 \mathrm{~cm}$. La distance comprise entre les deux maxima d'amplitude do profondeurs respectivement égales à $13,7 \mathrm{~cm}$ et $9,2 \mathrm{~cm}$ est de $46 \mathrm{~cm}$ alor's que la demi-longueur d'onde pour la profondeur moyenne de $11,6 \mathrm{~cm}$ supposée constante serail de $48 \mathrm{~cm}$. Le phénomène qui existe devant la plage est donc non pas une houle, mais un clapotis partiel, le coefficient de réflexion, facile à déterminer, étant de l'ordre de $16 \%$.

On peut apercevoir sur les photographies des figures 4 et 5 des crêtes secondaires, en particulier entre le rouleau déferlant et la crête principale qui la suit. On pourrait être tenté de penser que ces crêtes sont dues à la réflexion; mais on ne décele pas de propagation nette par rapport à la crête principale, comme ce serait le cas pour une onde réfléchie ou un harmonique de la houle incidente. Peut-être s'agit-il d'un autre phénomène propre à la houle en faible profondeur, tel que la crête secondaire qui résulte des calculs de M. Miche (1), p. 58 - et qu'il considérait dans son article comme limitant le domaine de validité de sa théorie.

Pour la houle II, le domaine à l'intérieur du- quel on peul mesurer lamplitude est trop faible pour qu'on puisse recomnaitre une ondulation due à la réflexion; s'il en existail une toutefois, la distance entre une crête et un creux serait bien supérieure à la demi-longueur d'onde. On peut donc penser que le coefficient de réflexion est moindre dans le cas des fortes cambrures, ce qui d'ailleur's semble conforme à certaines idées théoriques.

Pour les deux houles, on constate que le point de déferlement, tel que nous l'avons défini, ne coincide pas avec le maximum d'amplitude, mais le suit. Toutefois, il faudrait se garder de tirer une conclusion générale de deux essais.

\section{c) Surélévation du niveau moyen.}

Les images successives du film permettent de suivre l'évolution du profil de la houle dans la zone de déferlement. L'énergie se concentre de plus en plus à la crête; la crête augmente de plus en plus de hauteur par rapport au niveau de l'eau au repos et lor'squ'on passe le film, on voit, surtout pour la houle I, la crête faire un veritable saut avant son écroulement; on se rend compte également de ce phínomìne sur les courbes de la figure 12 .

Cés courbes nous permettent également de déterminer la surélévation du niveau moyen.

Elle est donnce par la figure 14 .

On peut aussi calculer le rapport de la hauteur de crête situéc au-dessus de niveau de l'eau au repos à la demi-amplitude au point de déferlement.
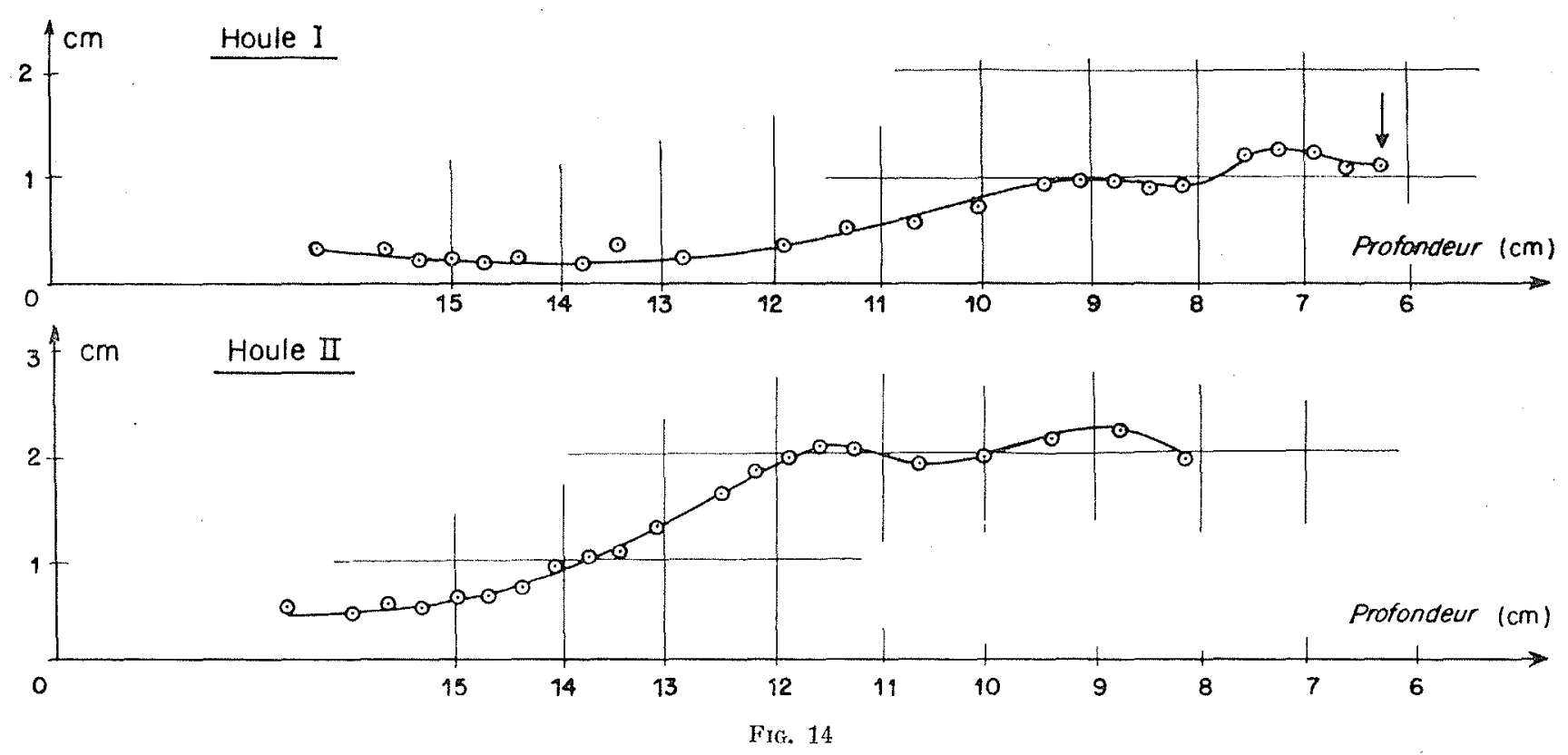

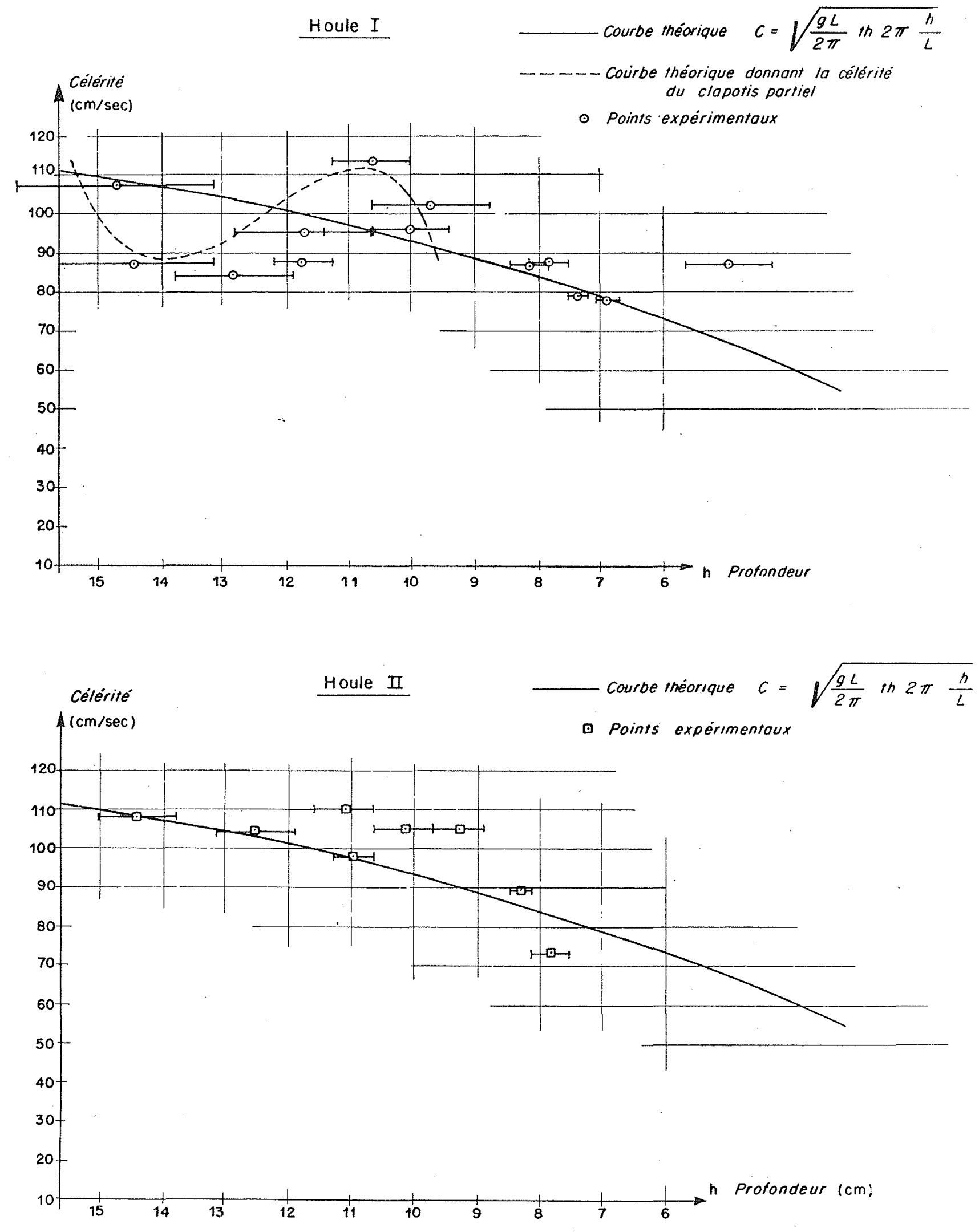

Fra. 15 
On trouve ainsi :

Critère de l'éboulement des particules:

$$
\begin{array}{ccc}
\text { Houle } & \text { I } \ldots \ldots \ldots \ldots & 1,45 \\
\text { Houle } & \text { II } \ldots \ldots \ldots \ldots & 1,48
\end{array}
$$

Critire de la tangente maximum:

$$
\begin{aligned}
& \text { Houle I . . . . . . . 1,40 } \\
& \text { Houle II . . . . . . } 1,34
\end{aligned}
$$

Ces chiffres sont très voisins de ceux indiqués par M. Larras (4).

\section{d) Célérité}

Nous avons essayé de suivre la variation de la célérité, - done de la longueur d'onde - de la houle le long de la pente. Pour cela, nous mesurions la distance parcourue par une crête pendant le temps séparant 10,$15 ; 20$ ou 25 images successives du film. Connaissant la vitesse à laquelle le film avait été pris, on en déduisait la célérité, considérée comme la vitesse de déplacement d'une crête.

Ce procédé est sujet à un certain nombre d'erreurs. Tout d'abord, la vitesse à laquelle le film a été pris -... qui étail d'ailleurs rigoureusement constante -..- ne peut ètre connue avee une grande précision; nous avons fait cependant un contrôle en comptant le nombre d'images séparant une période, e'est-à-dire séparant deux « situations $\gg$ de la houle, identiques ou du moins très voisines; l'incertitude est de 4 ou 5 images au maximum sur 140. Ensuite, quand la houle est très peu cambrée, il est difficile de localiser le sommet de la crête. Enfin, comme pour toule mesure, il existe une erreur lorsqu'on lit les longueurs sur l'écran de l'appareil de lecture de microfilms. L'erreur sur la mesure de la période est, par contre, pratiquement négligeable.

La deuxième erreur varie avec la cambrure de la houle; elle est d'autant plus petite que la houle est plus « aiguë», c'est-ì-dire pour une houle donnée, que la profondeur est plus faible. On voit done que la précision est meilleure pou: la houle II que pour la houle I et pour la houle I elle est d'autant meilleure que la profondeur est plus faible.

Malgré ces causes d'erreur et l'arbitraire qui règne dans le choix des intervalles de longueul. élémentaires servant à la mesure, nous donnons sur les graphiques de la figure 15 la répartition de la célérité avec la profondeur. Le point expérimental est le milieu d'un segment horizontal dont les extrémités ont mêmes profondeurs que les extrémités de l'intervalle de longueur élémentaire. Nous avons également porté sur ce mème graphique la courbe donnée par la formule :

$$
\mathrm{C}=\sqrt{\frac{g \mathrm{~L}}{2 \pi}} \text { th } \frac{2 \pi h}{\mathrm{~L}}
$$

( $L$ étant la longueur d'onde pour la profondeur h.)

On y voit que pour la houle I, les points sont très dispersés. Ce fait n'est peut-être pas entièrement dû aux erreurs de mesure, mais peut etre attribué en partie à l'existence du clapotis partiel; on sait en effet que, dans ce cas, si l'on suit une crète, sa vitesse de propagation varie dans le temps, tout en restant égale en moyenne à la valeur donnée par la formule précédente, au premier ordre tout au moins; par contre, en un point donné, le phénomène étant permanent, la célérité est constante, mais elle varie d'un point à un autre. Nous avons done porté sur la figure la courbe donnant la célérité d'un clapotis partiel en prenant pour coefficient de réflexion celui qui résulte de la figure 13 . On peut ainsi tracer la courbe en pointillé de la figure 15 . On voit que les points expérimentaux se répartissent autour de cette courbe; en particulier, les points expérimentaux extrêmes sont voisins du maximum et du minimum de la courbe.

Pour la houle II, les points sont un pen mieux groupés dans la zone qui précède le déferlement; ceci est dù, pour une bonne part, à ce que, la houle étant plus cambrée au large, les mesures de déplacement de crêtes sont plus faciles à faire, mais on peut penser aussi que cest parce que le coefficient de réflexion est sans doute plusfable.

On ne saurait d'ailleurs tirer de ces graphiques aucune conclusion quantitative précise sur la valeur de la formule :

$$
\mathrm{C}=\sqrt{\frac{g \mathrm{~L}}{2 \pi}} \text { th } 2 \pi \frac{h}{\mathrm{~L}}
$$

pour la célérité de la houle en profondeur décroissante, du fait des risques d'erreurs signalés plus haut.

\section{c) Rapport de l'amplitude is la profondenr an point de deferlement.}

M. Munk (3) a édifié une théorie du déferlement fondée sur la forme limite de l'onde solilaire; cette théorie ne s'applique que pour des cambrures au large suffisamment faibles. Elle est caractérisée par une valeur constante du rap-

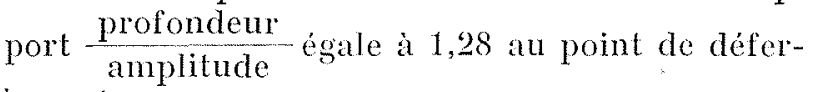
lement.

Il est intéressant de voir ce que domnent nos essais. On trouve ainsi :

Critère de l'éboulement des particules:

$$
\begin{array}{rrr}
\text { Houle } \quad \text { I . . . . . . . } & 1,34 \\
\text { Houle II . . . . . . } & 1,23
\end{array}
$$




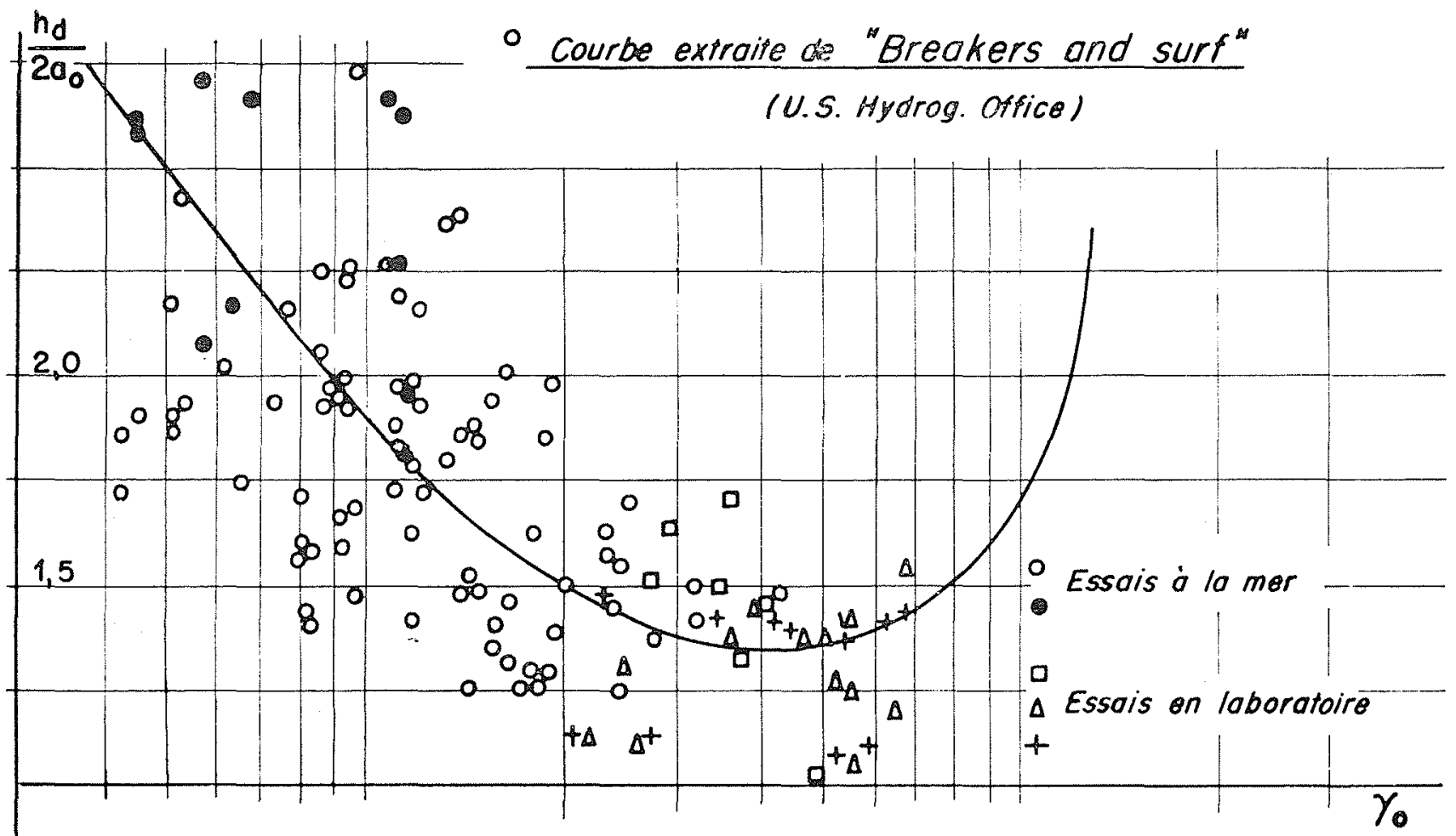

Résultats obtenus au Laboratoire Dauphinois d'Hydraulique

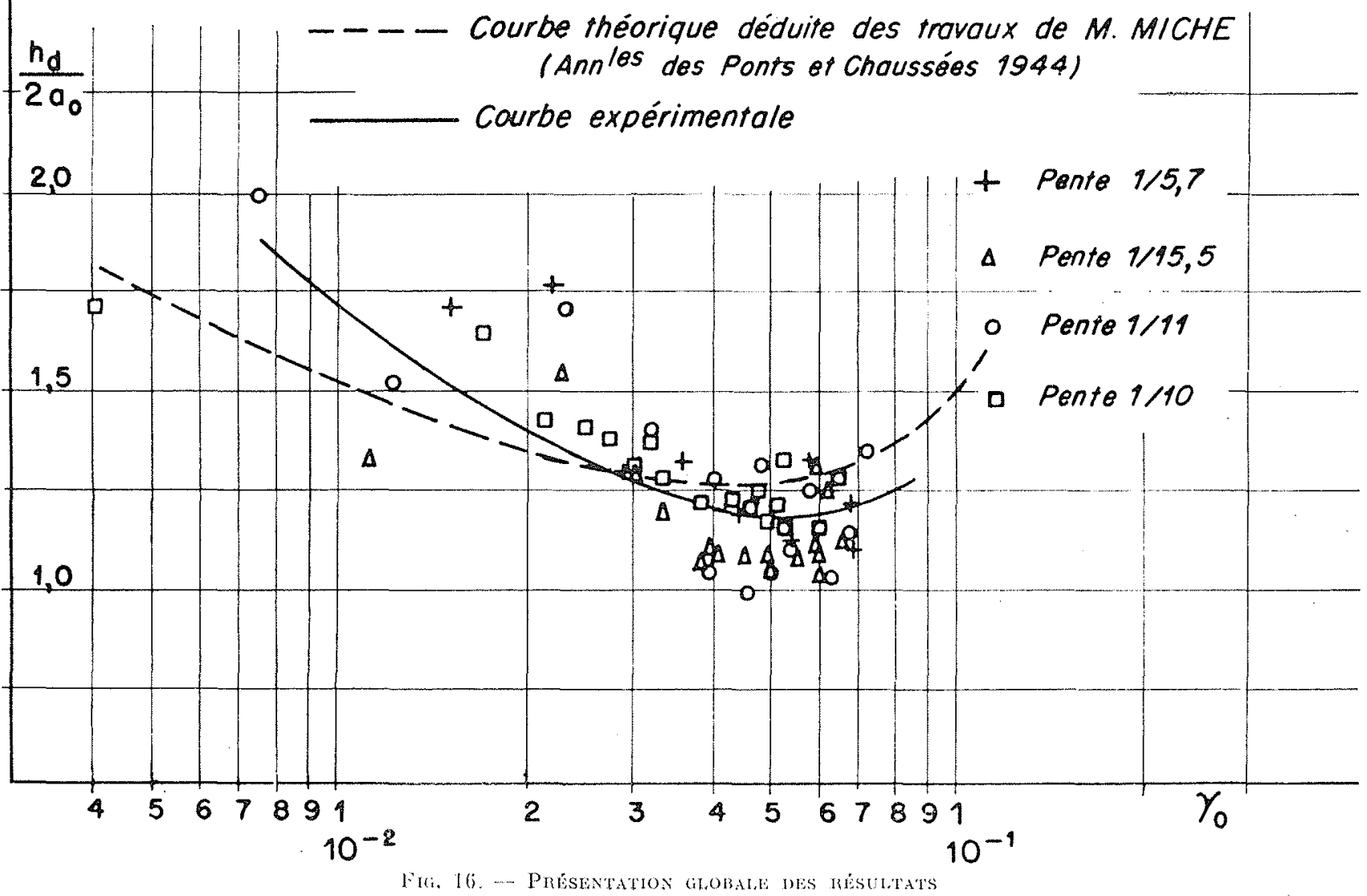


Critère de la tangente maximum :

$$
\begin{array}{lrr}
\text { Houle } & \text { I } \ldots \ldots \ldots \ldots & 1,28 \\
\text { Houle } & \text { II } \ldots \ldots \ldots \ldots & 1,21
\end{array}
$$

On voit ainsi que pour la houle I dont la cambrure au large n'est pas très élevée, la valeur de ce rapport est voisine de celle indiquée par les auteurs de la théorie en question.

\section{IV. - Présentation globale des résultats.}

Comme il est dit plus haut, nous avons rassemblé sur un graphique unique (fig. 16) donnant $\frac{k_{\ell}}{2 a_{0}}$ en fonction de $\gamma_{0}$, les résultats des essais $1,2,3,7$.

Nous y avons joint également une courbe expérimentale analogue extraite de « Breakers and surf $\gg(6)$. Nous ne prétendons point que nos résultats soient plus précis;-.il faut en effet considérer que les points portés sur le graphique de «Breakers and surf » ont été obtenus dans des conditions très variables; certains résultent d'observations à la mer, les autres ont été obtenus par diver's laboratoires. Il ne faut done pas s'étonner que la dispersion soit assez grande, surtout lorsqu'on connait les difficultés de l'observation. Quoi qu'il en soit, l'allure des courbes est analogue, mais la nôtre est au-dessous de la courbe américaine. Ceci peut s'expliquer de la façon suivante : le critère adopté dans « Breakers and surf » semble être l'apparition de la singularité anguleuse en crête qui précède dans le temps celui de la tangente verticale ou de la pente maximum.

Nous avons également porté sur notre graphique une courbe déduite de l'article de M. MICHE (1). Dans cet article, M. Miche étudie le déferlement de la houle en eau peu profonde en employant la théorie énergétique (donc en négligeant l'inftuence de la pente) et en faisant une approximation qui consiste essentiellement à confondre $t h \frac{2 \pi h}{\mathrm{~L}}$ avec $\frac{2 \pi h}{\mathrm{~L}}$. II indique également qu'on pourrait utiliser les équations exactes de la théorie énergétique, sans faire cette approximation. Nous avons fait ce calcul et l'allure de la courbe ainsi obtenue étant voisine de celle de la courbe expérimentale, nous avons cru bon de la faire figurer sur ce graphique. On voit que pour des cambrures comprises entre $3 \%$ et $7 \%$ où nos résultats sont bien groupés, la courbe expérimentale est légèrement décalée vers le bas par rapport à la courbe théorique; peut-être, est-ce également parce que le critère adopté par cette dernière est celui de la crête anguleuse.

\section{CONCLUSIONS}

Ces premiers essais ont déjà permis d'étudier essentiellement la profondeur de déferlement de Ia houle; ils nous ont renseigné également sur l'évolution du profil de la houle au voisinage du déferlement et sur certaines de ses caractéristiques telles que l'amplitude et la célérité. Nous avons pu préciser ainsi la notion de critère de déferlement. Nous avons étudié l'influence de différents facteurs tels que la pente - et très sommairement la rugosité - sur la position du point de déferlement et le profil de la houle dans cette zone. Pour une houle de cambrure au large donnée, la profondeur de déferlement est d'autant plus grande que la pente est plus forte au moins dans le domaine que nous avons examiné. Il semble aussi que, pour une pente fai- ble, un fond rugueux déplace vers le rivage le point de déferlement.

Ces essais montrent l'intérêt des études plus poussées actuellement en cours au Laboratoire Dauphinois d'Hydraulique sur le déferlement de la houle et l'évolution de ses caractéristiques (célérité, amplitude, vitesse des particules, etc.), ainsi que sur les facteurs (pente, rugosité, décrochements, etc.), qui peuvent intervenir. Mais ils indiquent aussi que certaines theories approchées (théorie énergétique donnant l'amplitude, théorie analogue donnant la profondeur de déferlement, clapotis partiel etc.), peuvent, dans certains cas pratiques, voisins de ceux que nous avons envisagé, donner un ordre de grandeur sur quelques caractéristiques de la houle déferlante.

\section{BIBLIOGRAPHIE SOMMAIRE}

(1) MICHE. -.-. Mouvements ondulatoires de la mer en profondeur constante ou décroissante.

(Annales des Ponts et Chaussées, janvier-février, mars-avril, mai-juin, juillet-août 1944.)

(2) STOKER. - The formation of breakers and bores. (Communications on applied mathematics 1948 , vol - I, $\mathrm{n}^{\circ}$ 1.)
(3) MUNK. - The solitary wave theory and its application to surf problems.

Annals of the New York Academy of Sciences, vol. 5,13 mai 1949.)

(4) LARRAS. - Le déferlement des lames sur les jetées verticales.

(Annales des Ponts et Chaussies, V, mai 1937.) 
(5) FERlio. .... Contributo sperimentate allo studio del frangimento di onde propagantisi in massa liquida di profondita descrescente.

Alti del Reale Institulo fenelo di scienze Letlere ed Arli 1939.)

(6) U.S. IIYDROQRAPHIC OFFICL. -... Breakers and surf. Principles in forecasting.

(7) SUTHONs, - The forecastints of sea and swell waves.

(8) BIESEL. - Le fille à houle systeme Neyrpic. (La Houlle Blanche, $\mathrm{n}^{\circ} 3,1048$.)

(9) FOLSOM. - Amphibious craft and their chatrateristics

(Marine Neus, vol. XXXIX, oct. 1947.)
(10) BIGELOWV et EDMONSON. - W Wind waves at soa Breakers and surf.

(H.O., pub. no 602.)

(11) o'BRIEN. - The causes of plunging and spilling breakers.

(Balletin of the Beach Frosion Board, vol. 3, II" 3,1949 .)

(12) BIESEL. - Le filtre à houle.

(Commanication présentée alt Comilé de la S.H.F., le 4 juin 1949.)

(13) PUTNAM et JOHNSON. -... The dissipation of wave energy by bottom friction.

(Transactions of the American Geophysical Union, vol. $30, \mathrm{n}^{\circ}$ 1, f́evrier 1949.)

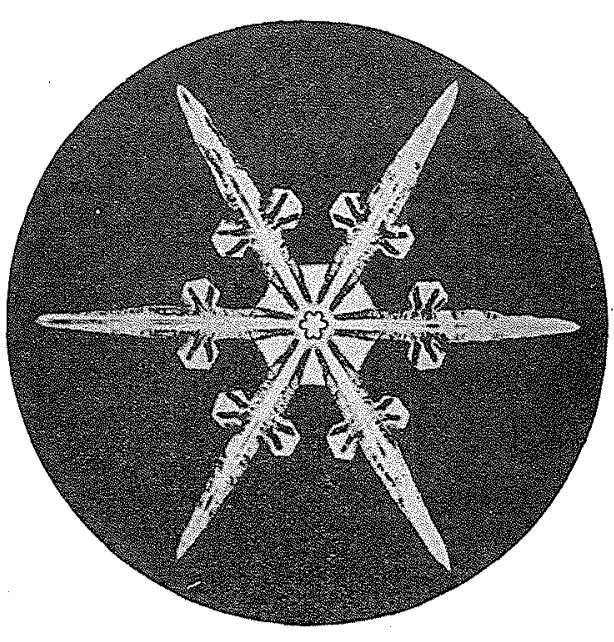

